\title{
Horizontal transfer of potato viroid PSTVd by Phytophthora infestans to and from host plants
}

\author{
Afanasenko O.S. ${ }^{1}{ }^{*}$, Khiutti A.V. ${ }^{1}$, Mironenko N.V. ${ }^{1}$, Lashina N.M. ${ }^{1}$, Matsushita Y. ${ }^{2}$ \\ ${ }^{1}$ All-Russian Institute of Plant Protection, St. Petersburg, Russia \\ ${ }^{2}$ NARO, Institute of Vegetable and Floriculture Science Tsukuba, Japan \\ * email: olga.s.afan@gmail.com
}

Potato spindle tuber viroid (PSTVd) is economically important pathogen and classified as high-risk plant quarantine disease. According to the European Plant Protection Organization (EPPO), the disease has been reported in 37 countries on all continents. The quarantine status of PSTVd and the possible significant losses of potato yield determine the importance of studies of various aspects of pathogen epidemiology. PSTVd is a single-stranded circular naked RNA genome, 356-363 nucleotides in length, lacking the ability to encode a protein and therefore being a parasite of the transcription mechanism of hosts. Replication of PSTVd is accompanied by the accumulation of viroid-derived small RNAs (vd-siRNAs) proposed to play a central role in disease symptom development. PSTVd is transmitted mechanical, by aphids, through pollen to progeny seeds and to other plants of potato and tomato. The aim of study was to identify a possible role of Phytophthora infestans in the transmission of PSTVd to potato and tomato plants. Three potato cultivars Gala, Riviera and Colomba were inoculated with strain PSTVd VP87. After 60 days post inoculation RT PCR diagnostic showed presence of PSTVd in these cultivars. Inoculation of PSTVd infected potato plants with $\mathrm{Ph}$. infestans isolate VZR18 was conducted. After the onset of late blight symptoms reisolation on rye agar of $\mathrm{Ph}$. infestans from infected plants was done. Positive diagnostic of PSTVd by RT-PCR in culture Ph. infestans after 1, 2, and 3 passages was showed. Two months after inoculation with $\mathrm{Ph}$. infestans isolate infected with PSTVd of healthy tomato plants RT-PCR revealed a 360-bp amplification product, indicating successful infection of plants. This is the first proof of horizontal transfer of PSTVd by Phytophthora infestans to and from host plants.

Acknowledgments: The research was supported by a grant from the Russian Science Foundation No. 20-46-07001. 